\title{
Non-traumatic nasal septal abscess in an immunocompetent patient*
}

\author{
Badar Salam and Andrew Camilleri \\ Department of ENT, South Manchester University Hospital, Wythenshawe, M23-9LT, United Kingdom
}

\begin{abstract}
SUMMARY
Nasal septal abscess is an uncommon condition. Most commonly it is secondary to nasal trauma, which leads to haematoma, and subsequent abscess formation. There are other less common causes like sinusitis, dental infections and furunculosis. Non-traumatic nasal septal abscess has also been reported in immunocompromised individuals.

We report a case of non-traumatic, spontaneous nasal septal abscess, in a healthy immunocompetent patient with no evidence of sinusitis or other localized infections. Using Medline and Google.co.uk search applications, there has been one previous report of such a condition. We stress the importance of excluding nasal septal abscess in patients presenting with nasal obstruction especially with signs of toxaemia.
\end{abstract}

Key words: nasal septum, abscess, non traumatic

\section{CASE REPORT}

A 38 years-old lady was referred to open access clinic by her family physician, with a four days history of painful, swollen, erythematous and stuffy nose. Oral antibiotic did not improve her condition. There was no history of trauma, recent sinusitis, or dental infection. Her past medical history revealed arthritis affecting her knees; otherwise she was fit and healthy. Physical examination revealed the patient to be slightly febrile, also swollen and erythematous nose was noted. Anterior rhinoscopy showed a swollen, tender anterior nasal septum, yielding to probing. The postnasal space, oropharynx, oral cavity, neck and remaining nasal examination using endoscope were normal. Her white blood count was raised to $14.7 \times 10^{9} / 1$ and neutrophil count to $12.59 \times 10^{9} / 1$. Platelets were 267 . She was commenced on intravenous Augmentin $1.2 \mathrm{~g}$, three times a day. The abscess was drained under GA. Nasal septum was found to be intact, a drain was sutured in the abscess cavity, and both nasal fossae were packed with BIPP. Her nasal swelling, erythema and congestion improved over the next 48 hours, packs and drain were removed then. The patient was discharged home on the third day, with oral Augmentin for a further five days. On review appointment, 10 days later her nasal mucosa had healed well except for slight oedema. There was good septal support with no cosmetic deformity.

\section{DISCUSSION}

Nasal septal abscess is defined as a collection of purulent material between the cartilaginous or bony septum and overlying mucoperichondrium or mucoperiostium ${ }^{(1)}$. It is a rare entity. Nasal septal abscess was first reported nearly one hundred years ago by Arnal in Paris. Arnal reports of "Falls, beats on the nose, nasal infections and violent actions by foreign bodies in the nasal cavity" as potential etiologies. Nearly $75 \%{ }^{(1)}$ of septal abscesses are secondary to trauma. Less frequently nasal septal abscess occurs following nasal surgery. Other reported cases are due to furunculosis ${ }^{(2)}$ of the nasal vestibule, dental disease and extractions ${ }^{(3)}$, influenza, acute sphenoiditis ${ }^{(4)}$ and acute ethmoiditis. Non-traumatic nasal septal abscess has also been reported in immunocompromised patients ${ }^{(5)}$. There has been one previous report of non-traumatic, spontaneous nasal septal abscess in an immunocompetent patient ${ }^{(6)}$. Septal abscess usually presents as bilateral nasal obstruction due to tender, swollen anterior nasal septum and associated symptoms of toxaemia, such as pyrexia, raised pulse rate, headache and malaise. Commonest organisms cultured from septal abscesses are Staphylococcus aureus, S. epidermidis, Streptococcus pneumonia, S. milleri, S. viridians, Haemophilus influenza and anaerobic organisms ${ }^{(7)}$. Late diagnosis and treatment is associated with increased incidence of serious complications. Rich perineural lymphatics at the anterior skull base and valve less venus communications between the angular vein and cavernous sinus through the ophthalmic veins can facilitate the spread of infection, leading to cavernous sinus thrombosis, meningitis ${ }^{(8)}$, brain abscess and subarachnoid empyema ${ }^{(9)}$. Contiguous spread of infection can also cause orbital cellulitis and abscess. Since the septal cartilage is avascular, deriving its nourishment from overlying mucoperichondrium, the stripping of mucoperichondrium that occurs in abscess formation can cause ischemic necrosis and subsequent septal perforation resulting in a saddle deformity. In children 
this can result in abnormalities of nasal and facial growth. Prompt diagnosis and treatment is of paramount importance. Incision and drainage of the abscess as well as broad-spectrum intravenous antibiotic, to include anaerobic cover is advised. The aspirate is sent for culture and sensitivity. A soft drain is inserted in the abscess cavity and nose is packed bilaterally for 48 hours, to prevent re accumulation. Patients are generally discharged with 5-7 days course of oral antibiotic, adjusted to the results of the culture and sensitivity.

\section{CONCLUSION}

Nasal septal abscess is a rare entity, if treated promptly it can be cured. However any delay in the treatment can lead to catastrophic complications. We would like to emphasize the importance of being attentive to the possible presence of nasal septal abscess in a patient complaining of nasal obstruction.

\section{REFERENCES}

1. Ambrus PS, Eavey RD, Sullivan BA, Wilson WR, Kelly JH. Management of nasal septal abscess. Laryngoscope 1981; 91: 575582.

2. Larchenko RM. On abscesses of the nasal septum in children. Vestniko Oto-Rhino-Laringologii 1961; 23: 46-49.

3. da Silva M, Helman J, Elicher I, Joachims H. Nasal septal abscess of dental origin. Arch Otolaryngol 1982; 108: 380-381

4. Collins MP. Abscess of nasal septum complicating isolated acute sphenoiditis. J Laryngol Otol 1985; 99: 715-719.
5. Saurabh BS, Andrew HM, Kelvin CL. Non traumatic nasal septal abscesses in immunocompromised. Etiology, Recognition, Treatment, and Sequelae. Am J Rhinol 2000; 14: 39-43.

6. Bennett JDC, Rapado F. Nasal septal abscess in a healthy, nonimmunocompromised patient. Hospital Med 1998; 59: 78.

7. Cantey PA, Berkowitz RG. Haematoma and abscess of the nasal septum in children. Archives of Otorhinolaryngology 1996; 122: 1373-1376.

8. Eavey RD, Malekzakeh M, Wright HT Jr. Bacterial meningitis secondary to abscess of the nasal septum. Paediatrics 1977; 60: 102-104.

9. McCaskey CH. Rhinogenic brain abscess. Laryngoscope 1951; 61: 460-467.
B Salam

5 Mallory court

Belgrave road

Bowdon

Altrincham - Cheshire

WA14-2NP

United Kingdom

Tel: +44-161-924 0145

Fax: +44-787-968 6224

E-mail badariram@hotmail.com 